\title{
Mapping transmission foci to eliminate malaria in the People's Republic of China, 2010-2015: a retrospective analysis
}

Jun Feng, Hong Tu, Li Zhang, Shaosen Zhang, Shan Jiang, Zhigui Xia and Shuisen Zhou*

\begin{abstract}
Background: China has initiated the National Malaria Elimination Action Plan, which aims to eliminate malaria by 2020. However, the transmission of malaria occurs sporadically or in distinct foci, which greatly hampers progress toward elimination in China and other countries. The object of this study was to foci categorization and evaluates whether the response met the requirements issued by the nation or WHO.

Methods: Residual transmissions were investigated and located with fine spatial resolution mapping from parasitological confirmed malaria cases by use of routine national surveillance data. The "1-3-7" timeframes were monitored for each focus between 2012 and 2015. Each focus was identified, and the application of appropriate measures was evaluated.
\end{abstract}

Results: A total of 5996 indigenous cases were recorded between 2010 and 2015; during this period, the number of cases declined by $99.1 \%(2010, n=4262 ; 2015, n=39)$. Most indigenous cases (92.5\%) were reported in Anhui $(n=2326)$, Yunnan ( $n=1373)$, Henan $(n=930)$, Hubei $(n=459)$, and Guizhou $(n=458)$. The temporal distribution showed that the indigenous malaria cases were clustered during the period of May to August. A total of 320 foci were carefully investigated and analyzed: 24 were active foci; 72, residual non-active foci; and 224 cleared-up foci. For the foci response evaluation, all the active foci were investigated within 7 days, while $80.2 \%$ of the residual non-active foci were responded within 7 days. In addition, reactive case detection (RACD) was carried out with $92.9 \%$ of the active foci and vector investigation carried out with $75 \%$. For residual non-active foci, RACD was carried out with $83.2 \%$ and vector investigation with $78.2 \%$ of the foci.

Conclusions: This study used nationwide data to categorize foci in China and evaluate the response of these areas during the control and elimination phases. Our approach stratifies future control responses by identifying those locations where the elimination of endemic transmission is needed, such as in the counties at the China-Myanmar border and in Tibet. In addition, this study will help local CDC staff to reassess their needs and responses against different types of foci during the elimination and post-elimination phases.

Keywords: Malaria elimination, Foci, China

\footnotetext{
*Correspondence: shuisenzhou@126.com; zss163@hotmail.com

National Institute of Parasitic Diseases, Chinese Center for Disease Control and Prevention; Key Laboratory of Parasite and Vector Biology, Ministry of Health; WHO Collaborating Centre for Tropical Diseases; National Center for International Research on Tropical Diseases, Shanghai 200025, People's Republic of China
} 


\section{Background}

China initiated the National Malaria Elimination Action Plan (NMEAP) in 2010, which aimed to eliminate indigenous malaria in non-border areas by the end of 2015, and eliminate it nationwide by the end of 2020 [1]. All the counties in China were classified into 4 types, and each type has its own strategy and interventions [2] (Table 1).

To achieve the national goal of malaria elimination, China has made great strides in controlling indigenous malaria over the past several decades [3]. After implementing an integrated control strategy and interventions, along with socio-economic development, urbanization progress, and changes in the natural environment and malaria vectors, the incidence of indigenous malaria in China has sharply declined, and the malariaendemic areas have dramatically shrunk [4]. Since 2010, transmissions mainly occurred in the counties along the China-Myanmar border and in Motuo County of the Tibetan Autonomous Region of China.

Malaria tends to persist in "foci" or localized areas of self-sustaining transmission, due to spatially heterogeneous influences, such as social and ecological factors that include Anopheline mosquito density, agricultural practices, human behavior, wealth, education, access to and utilization of health care, and urbanization [5]. In the elimination phase, cases occur sporadically or in distinct foci. In our investigation, the foci were classified into 3 types (active, residual non-active and cleared-up) according to the guidelines issued by the World Health Organization (WHO) [6]. The elimination of residual foci is a dynamic process, taking place mainly during the late stage of malaria elimination.

Monitoring the status of foci, with the precise identification of their functional status, is the cornerstone for success in interrupting malaria transmission and preventing the re-introduction of malaria where potential foci (foci with imported cases but without proof of local transmission) may be present. This step was important for China because there were many areas where transmission was sharply reduced but the vectors still exist.
To interrupt malaria transmission, a two-pronged approach is required: disease management (alleviation of symptoms and prevention of biting by mosquitoes) and disease prevention through vector control.

The object of this study is to categorize foci by using country-wide data collected under operational conditions during the important period from the control phase to the elimination phase in China, and to evaluate whether the responses meet the requirements set out by national or WHO guidelines. Many factors would be challenges in the performance of the case investigation, foci investigation and response. The findings are likely to reflect the real situation and would help local CDC staff to reassess their needs and responses against different types of foci during the elimination and postelimination phases.

\section{Methods}

\section{Study design}

A retrospective study was conducted to explore malariaendemic characteristics from January 1, 2010 to December 31, 2015 at the Chinese Center for Disease Control and Prevention (CDC) [7-12]. All individual cases from the Infectious Diseases Information Reporting Management System (IDIRMS, http://chinacdc.cn) were carefully reviewed and analyzed [13]. The IDIRMS, which was set up in 2004 after the SARS outbreak, is a standardized platform that provides health care systems nationwide the ability to detect, analyze, prevent, and respond to any communicable disease in the country. The data for the study were selected by use of the reporting data and reporting area, but the data from Hong Kong, Macao, and Taiwan were excluded from these statistics. The IDIRMS parameters consisted of the geographical distribution, species composition, gender, and age distribution of the cases. Both clinically diagnosed cases and laboratory-confirmed cases were included in this analysis (Table 2).

Another reporting system, the Parasitic Diseases Information Reporting Management System (PDIRMS, http://chinacdc.cn), was set up in 2012 to report on 3

Table 1 Classification of county types and strategy implemented according to NMEAP

\begin{tabular}{lll}
\hline Type & Classification & Strategy \\
\hline I & $\begin{array}{l}\text { Local infections are detected in } 3 \text { consecutive years, and } \\
\text { the incident rate is equal to or greater than } 1 \text { in } 10,000 .\end{array}$ & $\begin{array}{l}\text { The integrated interventions of case management and } \\
\text { vector control will be scaled up to reduce disease } \\
\text { incidence because annual parasite incidence (API) }<1 \\
\text { compared to } 2010 .\end{array}$ \\
II & $\begin{array}{l}\text { Local infections are detected in } 3 \text { consecutive years, and } \\
\text { the incident rate is lower than } 1 \text { in } 10,000 \text { in at least one } \\
\text { of those } 3 \text { years. }\end{array}$ & $\begin{array}{l}\text { Response to any possible malaria cases and active foci is } \\
\text { the main strategy to interrupt local transmissions. }\end{array}$ \\
No local infections reported for 3 years. & Enhance monitoring and surveillance of imported cases to \\
Areas without malaria epidemic. & Senst secondary transmission. \\
& & cases.
\end{tabular}


Table 2 Definitions used in this study

\begin{tabular}{ll}
\hline Type of malaria & Description \\
\hline Clinically diagnosed case & An individual with malaria-related \\
& symptoms (fever [axillary \\
& temperature $\left.\geq 37.5^{\circ} \mathrm{C}\right]$, chills, severe \\
& malaise, headache, or vomiting) at \\
& the time of examination. \\
& A clinical case confirmed by \\
Laboratory-diagnosed case & microscopy, polymerase chain \\
& reaction, or rapid diagnostic tests in \\
& the laboratory. \\
& A case contracted locally with no \\
Indigenous case & evidence of importation and no \\
& direct link to transmission from an \\
& imported case. In this study, an \\
& indigenous case refers to malaria \\
acquired by mosquito transmission & in China.
\end{tabular}

Imported case

A malaria case or infection in which the infection was acquired outside the area in which it was diagnosed. Here, it refers to the patient who acquired the illness from a known malaria-prevalent region outside China.

Induced case

Introduced case

Recrudescent case

Focus

A case in which the origin of the illness can be traced to a blood transfusion or other form of parenteral inoculation of the parasite but not to transmission by a natural mosquito-borne inoculation.

A case contracted locally, with strong epidemiological evidence linking it directly to a known imported case (first-generation local transmission).

Recurrence of asexual parasitemia of the same genotype(s) that caused the original illness, due to incomplete clearance of asexual parasites after antimalarial treatment.

Death from malaria

Patient with signs and symptoms of complicated malaria, with confirmed diagnosis of $P$. falciparum (or $P$. vivax) or associated infection.

A defined and circumscribed area situated in a currently or formerly malarious area that contains the epidemiological and ecological factors necessary for malaria transmission.

Reactive case detection

A process that involves an active response after the identification of a local or imported case in a receptive area where the transmission intensity is low or assumed to be interrupted.

diseases: malaria, schistosomiasis, and echinococcosis. Malaria was monitored and reported on within the time frame indicators using the "1-3-7" strategy. The "1-3-7" strategy, which refers to the reporting of malaria cases within 1 day, case confirmation and investigation within 3 days, and foci investigation and response to prevent further transmission within 7 days, was launched in 2012 to guide and monitor the elimination process nationwide [14]. Each case was investigated by staff at the county CDC to classify it as indigenous or imported; the definitions appear in Table 2 [15]. The PDIRMS contains the date of diagnosis, date of reporting, date of the case investigation, case classification (indigenous, imported, or other [induced, introduced, or recrudesce]), focus investigation, and foci response, such as reactive case detection (RACD) and indoor residual spraying (IRS). Both the website for the IDIRMS and the PDIRMS are private. Malaria department in NIPD was responsible for national malaria data for these two systems and have permission to access them.

\section{Data extraction for foci identification and response evaluation}

In this study, a natural village is considered the smallest unit of focus. "Active focus" refers to a focus with ongoing transmission; "residual non-active focus" refers to a transmission that was interrupted recently (1-3 years ago). A "cleared-up focus" is defined as a focus with no local transmission for more than 3 years.

For foci identification, the data of the indigenous cases from 2012 to 2015 in the IDIRMS were carefully reviewed and analyzed. Because it was not possible to distinguish the precise foci location for domestically mobile cases within the country (these cases are also defined as indigenous cases), only the foci with exact information were selected for categorization. If 2 or more cases were reported at 1 focus, they were considered and recorded as only one focus because the aim of this study is to classify foci and evaluate the response strategy. For the foci response evaluation, only the indigenous cases from the IDIRMS that matched the data in the PDIRMS were selected and analyzed. The population data for every county in China from 2010 to 2015 were obtained from the National Bureau of Statistics of China (http://data.stats.gov.cn/). All malaria cases reported were geo-coded and matched to the county-level layers of polygon and point using the software ArcGIS 10.1(Environmental Systems Research Institute, Inc., Redlands, CA).

\section{Results}

Indigenous malaria in China, 2010-2015

From 2010 to 2015,5996 indigenous cases were recorded by the IDIRMS. During this period, the reported indigenous cases sharply declined by $99.1 \%$ (2010, $n=$ $4262 ; 2015, n=40)$. Since data of indigenous malaria cases were not available for 2010-2011, the epidemiological data for Plasmodium species from 2012 to 2015 were collected. These data were analyzed, and the results indicated that most of the indigenous cases $(63.3 \%)$ were 
attributed to $P$. vivax. Like $P$. vivax, the number of local $P$. falciparum cases declined from 97 in 2010 to 1 in 2015 (this strain only occurred in Yunnan Province).

Transmission largely decreased during the study period; indigenous cases occurred in 303 counties in 18 provinces in 2010, while in 2015, transmissions occurred in only 9 counties in 4 provinces (Table 3 ).

In this study, indigenous cases occurred in $93.6 \%$ of the Type I and Type II counties. From 2010 to 2015, transmissions in Type I and Type II counties significantly decreased, particularly for Type II counties, while in 2015 only 1 Type II county in Dandong city, Liaoning Province, reported indigenous cases $(n=2$; Fig. 1$)$.

Most indigenous cases (92.5\%) were reported in Anhui $(n=2326,38.8 \%)$, Yunnan $(n=1373,22.9 \%)$, Henan $(n=$ 930, 15.5\%), Hubei $(n=459,7.7 \%)$, and Guizhou ( $n=$ 458, 7.6\%). However, in Liaoning and Hainan, where local transmission was blocked for 4 years, officials reported 2 local $P$. vivax and 6 local $P$. malariae cases in 2015 (Fig. 2).

\section{Temporal distribution of indigenous malaria}

The temporal distribution showed that indigenous malaria clustered between May and August. The highest number of transmissions was reported in June 2012, with 63 indigenous cases. The indigenous cases have sharply decreased since 2013: for example, only 12 indigenous cases were reported between May and August 2015, a $92.7 \%$ reduction compared with the same months in 2012 (Fig. 3). The residual auto correlation function (ACF) and residual partial correlation function (PACF) for the indigenous model also proved that the summer season (May-August) was the time frame with the most reported cases (Fig. 4).

\section{Foci identification and classification}

A total of 426 indigenous cases that were reported between 2012 and 2015 were carefully investigated and analyzed for foci identification and classification. Despite some unclear foci investigations $(n=60)$ and 2 or more cases reported in 1 focus $(n=46), 320$ foci were finally obtained and used for foci identification (Fig. 5).

The 320 foci were investigated and classified as 24 active foci, 72 residual non-active foci, and 224 cleared-up foci. The 24 active foci were distributed in 6 counties of Yunnan, 1 county of Hainan, 1 county of Liaoning, and 2 counties of Tibet (Table 4). The 72 residual non-active foci were distributed across 15 counties of Yunnan, 2 counties of Tibet, and 2 counties of Anhui.

\section{Foci response evaluation}

For the foci response evaluation, $43.9 \%(n=182)$ of the 426 indigenous cases selected from the IDIRMS were matched in the PDIRMS (Fig. 5). Further analysis found 32 of them were classified as 2 or more cases reported in 1 focus; therefore, 150 foci were evaluated for foci response.

In 2015, 28 indigenous cases were reported and foci response was carried out. All the active foci were investigated within 7 days; $92.9 \%(n=26)$ of foci carried out RACD, and $75 \%(n=21)$ carried out vector investigations (Table 5). Two positive patients were screened by RACD and found to have P. vivax (Yingjiang County) and P. malariae (Sanya County). A total of 101 residual non-active foci were evaluated, and most of them $(n=$ $81,80.2 \%$ ) responded within 7 days (Table 5). In addition, $83.2 \%(n=84)$ of the foci carried out RACD, and $78.2 \%(n=79)$ carried out vector investigations. Four people were diagnosed as malaria-positive by RACD, and $83.2 \%(n=84)$ of the foci carried out IRS. For the cleared-up foci, 21 foci were matched in both systems: only 1 focus investigated and responded within 7 days, while 16 carried out RACD and 19 conducted vector investigations (Table 5).

\section{Discussion}

During the elimination phase, population-level measures become inefficient and inadequate, so as countries

Table 3 Indigenous cases in China, 2010-2015

\begin{tabular}{llllllll}
\hline Year & Total cases & Indigenous (\%) & Local P. $v(\%)$ & Local $P . f(\%)$ & $\begin{array}{l}\text { No. of counties } \\
\text { with transmissions }\end{array}$ & $\begin{array}{l}\text { No. of Type } I^{a} \text { counties } \\
\text { with transmissions }\end{array}$ & $\begin{array}{l}\text { No. of Type } \|^{b} \text { counties } \\
\text { with transmissions }\end{array}$ \\
\hline 2010 & 7855 & $4262(54.3)$ & $N^{c}$ & $97(2.3)$ & 303 & 71 & 203 \\
2011 & 4498 & $1308(29.1)$ & $N^{c}$ & $32(2.4)$ & 155 & 60 & 92 \\
2012 & 2718 & $244(9.0)$ & $228(93.4)$ & $16(6.6)$ & 41 & 30 & 9 \\
2013 & 4128 & $86(2.1)$ & $77(89.5)$ & $9(10.5)$ & 12 & 9 & 3 \\
2014 & 3078 & $56(1.8)$ & $45(89.3)$ & $6(10.7)$ & 10 & 8 & 1 \\
2015 & 3116 & $39(1.2)$ & $38(97.5)$ & $1(2.5)$ & 9 & 187 & 1 \\
Total & 25,393 & $5995(23.5)$ & 393 & 32 & 530 & 309 & \\
\hline
\end{tabular}

aType I counties refer to areas with local infections that are detected in 3 consecutive years with an incidence rate equal to or greater than 1 in 10,000 ${ }^{\mathrm{b}}$ Type II counties refer to areas with local infections that are detected in 3 consecutive years with an incidence rate lower than 1 in 10,000 in at least 1 of those 3 years

${ }^{\mathrm{c}} \mathrm{NA}$ indicates that data were not available in the annual reporting system 


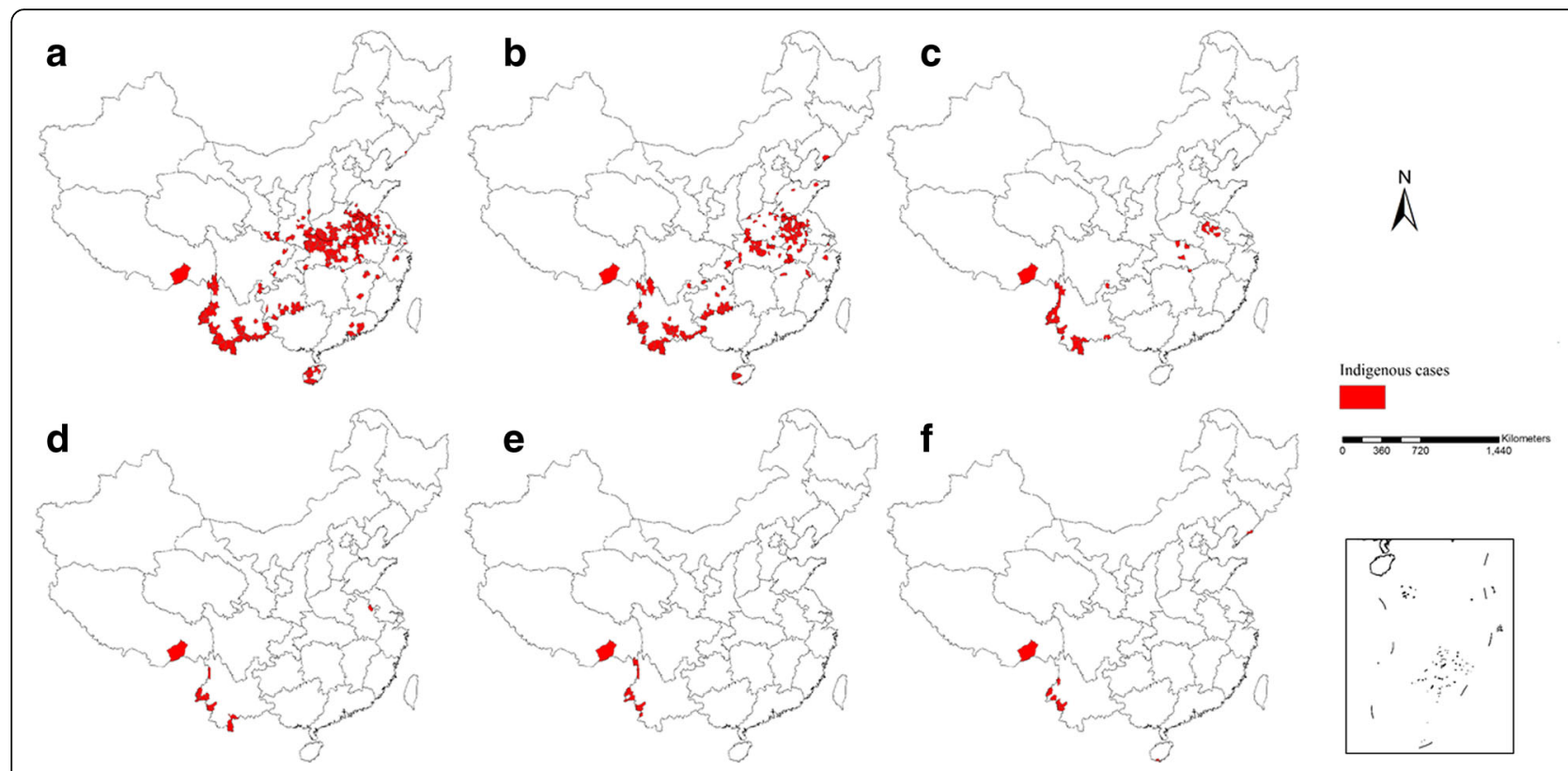

Fig. 1 Indigenous malaria in China, 2010-2015. a 2010, b 2011, c 2012, d 2013, e 2014, and f 2015. The red zones represent the areas where indigenous cases occur (county level)

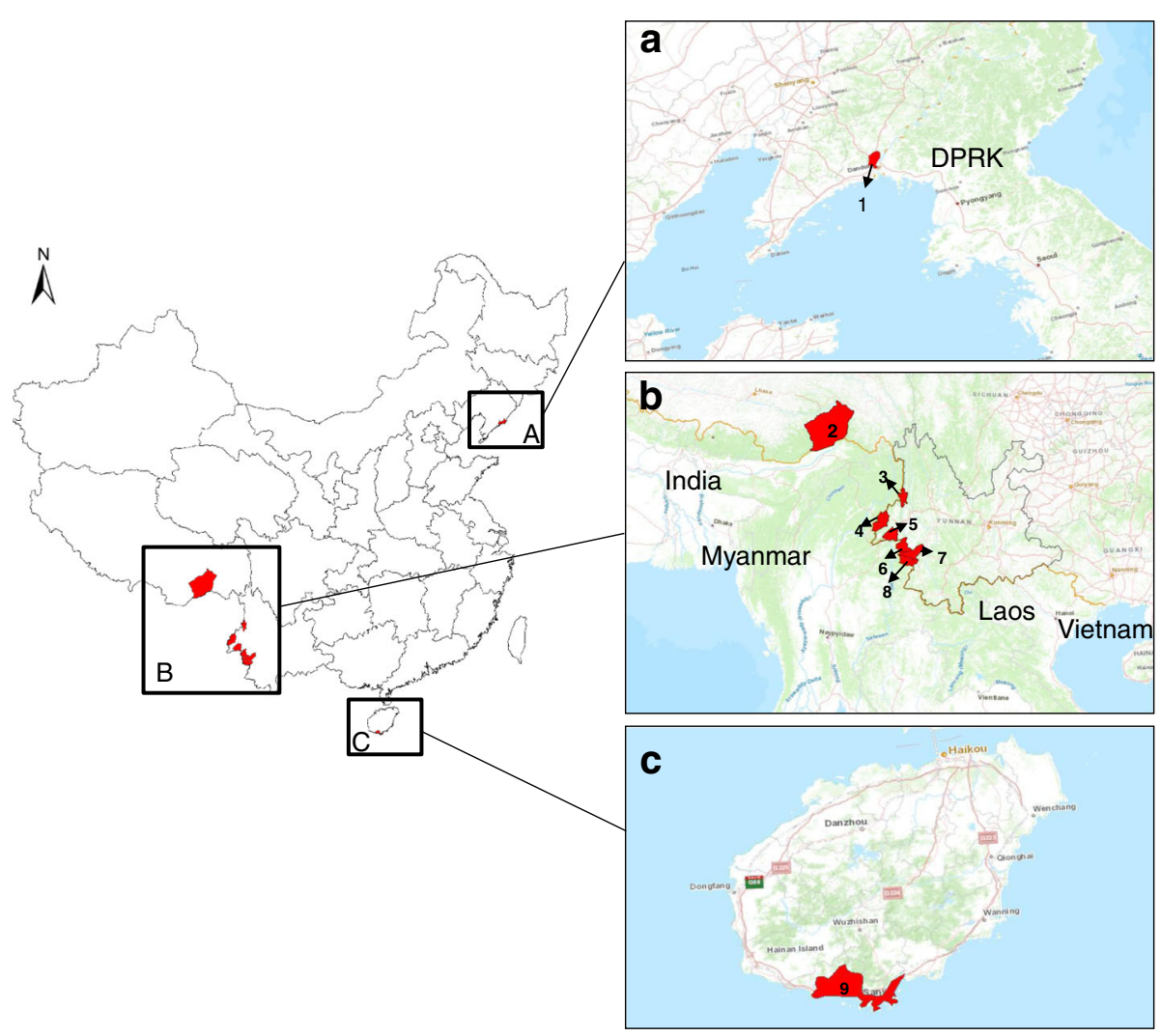

Fig. 2 The foci at county-level and its neighboring countries reported in 2015. a 1 represents Donggang County in Liaoning province neighboring with Democratic People's Republic of Korea (DRPK), b 2 represents Motuo County in Tibet, 3, 4, 5, 6, 7, 8 represent counties of Lushui, Yingjiang, Mangshi, Zhengkang, Gengma, Cangyuan in Yunnan province, respectively, where neighboring with Myanmar, c 9 represents Sanya County in Hainan province 


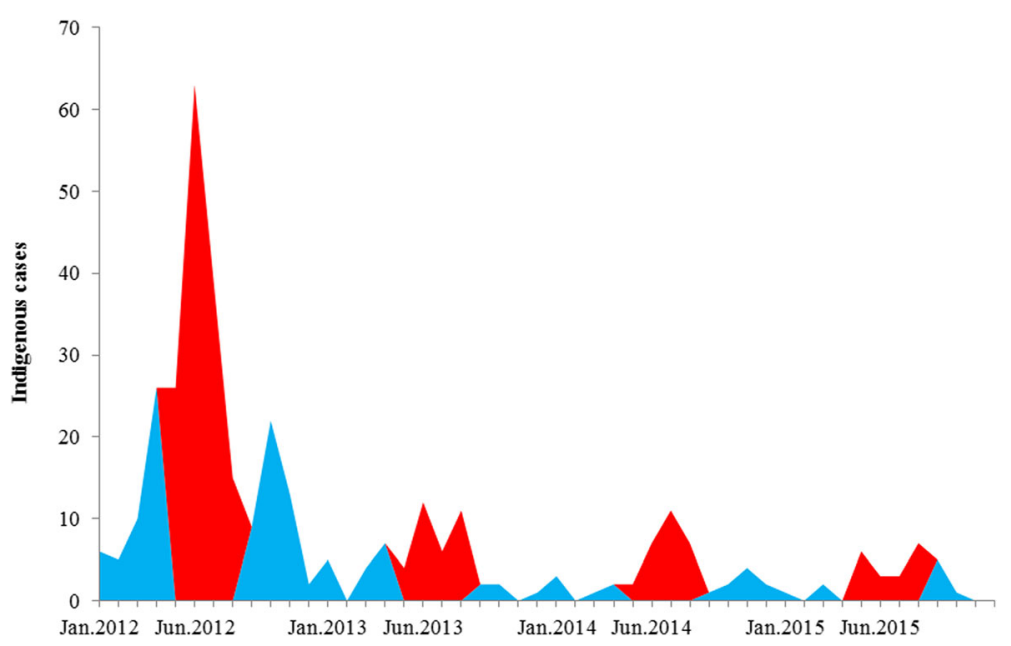

Fig. 3 Temporal distribution of indigenous malaria, 2012-2015. Red zones represent the period from May to August, and blue zones represent the period from September to April (the next year)

approach the goal of eliminating malaria, individualbased estimates of transmission must identify foci where resources should be targeted because transmission remains high [16]. Aggregate ratios of indigenous-toimported cases in time (or in space) alone, could obscure localized transmission if, for example, most cases failed to transmit but some pockets of transmission remain.

The border areas are a great challenge for malaria elimination in China because they harbor a mixed endemic region with both $P$. falciparum and $P$. vivax malaria transmission [17]. The natural environment in these areas is complex and a variety of malaria vectors, such as Anopheles dirus and An. minimus, usually co-exist in a single setting and have a high vector capacity for transmitting malaria [18]. In addition, there is a large mobile cross-border population since there is no natural barrier in this region, which makes management of imported malaria a significant challenge [19-21]. Poor transportation also makes it difficult to conduct epidemiological studies and blood smear verification within 3 days [22] In this study, the healthcare workers' response in these foci was inefficient, although all the cases at the foci were treated within 7 days. First, not all the active foci carried out RACD and IRS, which are required by the national strategy. Second, only three-quarters of the active foci conducted a vector investigation; without favorable entomological information, 2 potential risks remain.

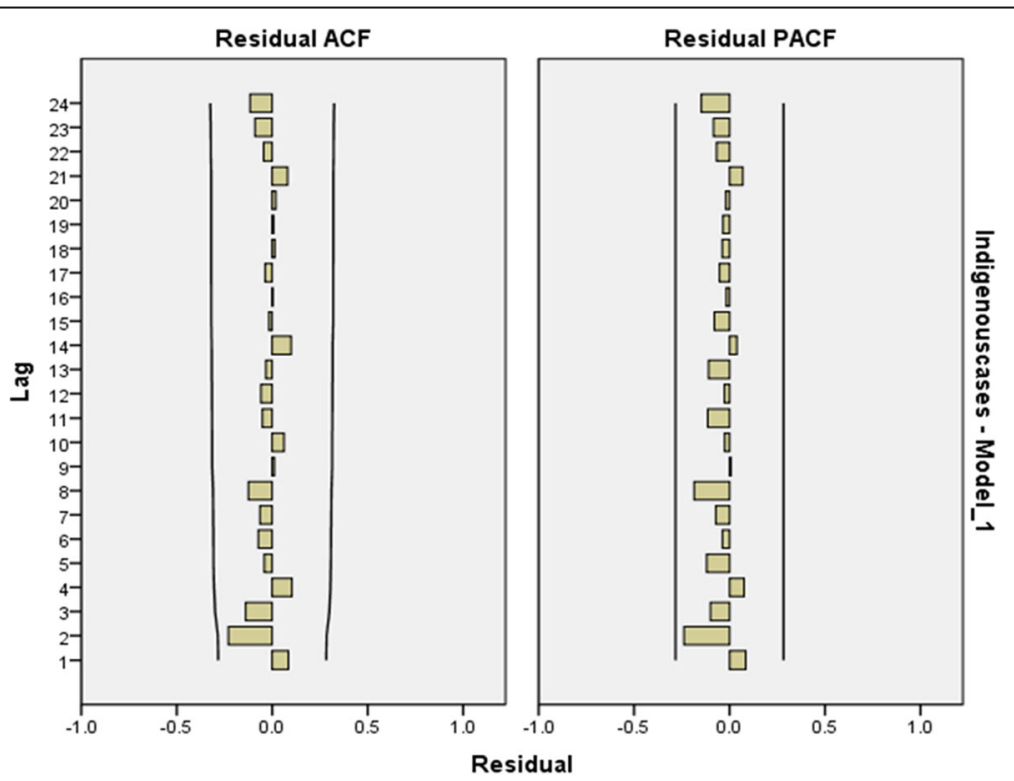

Fig. 4 Residual ACF and residual PACF of indigenous malaria, 2012-2015 


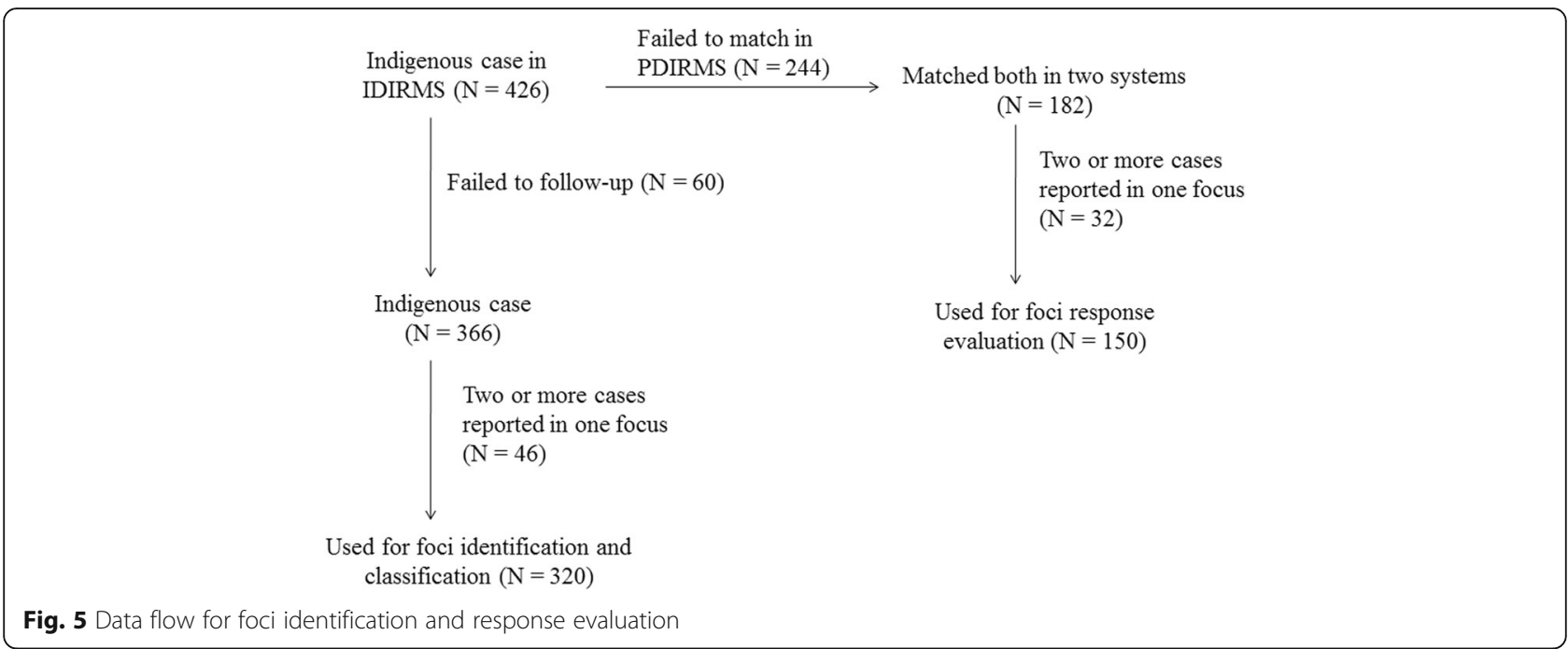

Table 4 Active foci in China (the foci that were investigated in each natural village ${ }^{a}$ )

\begin{tabular}{|c|c|c|c|c|c|}
\hline Foci No.' & Province & County & Township & Village & Case \\
\hline 1 & Yunnan & Yingjiang & Nabang & Jiedao & 2 \\
\hline 2 & & & & Nabang & 1 \\
\hline 3 & & & & Lishu & 1 \\
\hline 4 & & & & Daonong & 1 \\
\hline 5 & & & & Qiaotou & 1 \\
\hline 6 & & & Kachang & Caobei & 1 \\
\hline 7 & & Gengma & Mengding & Hanhong & 1 \\
\hline 8 & & & & Qiushan & 1 \\
\hline 9 & & Mangshi & Xuangan & Qincaitang & 1 \\
\hline 10 & & & Santaishan & Yunqian & 1 \\
\hline 11 & & Zhengkang & Mengdui & Yakou & 1 \\
\hline 12 & & Lushui & Pianma & Pianma & 1 \\
\hline 13 & & Canyuan & Banlao & Banlao & 1 \\
\hline 14 & Tibet & Motuo & Beibeng & Gelin & 1 \\
\hline 15 & & & & Jiangxin & 1 \\
\hline 16 & & & & Beibeng & 1 \\
\hline 17 & & & Motuo & Yadong & 1 \\
\hline 18 & & & & Bodong & 1 \\
\hline 19 & & & Dexing & Dexing & 1 \\
\hline 20 & & Linzhi & Bayi & Yingbin & 1 \\
\hline 21 & Hainan & Sanya & Fenghuang & Lixin & 4 \\
\hline 22 & & & & Baolong & 2 \\
\hline 23 & & & & Nandao & 1 \\
\hline 24 & Liaoning & Donggang & Jiangzi & Zhangdao & 2 \\
\hline
\end{tabular}

${ }^{\mathrm{a} A}$ natural village has approximately 50 households with a population of 200 to 250 people and is the lowest administrative level in China. In China, a natural village with a reported malaria case is considered a focus

${ }^{b}$ If 2 or more cases were reported at 1 focus, only 1 focus was recorded. In this study, more than 1 case was reported in Jiedao, Lixin, Baolong, and Zhangdao: therefore, those 4 sites were considered as 4 foci
One is that the imported $P$. vivax can be re-introduced under conducive ecological conditions (transmission season) if the area harbors an appropriate vector, although there is little evidence of re-introduction of imported malaria in China [23]. Another is the increasing importation of $P$. falciparum to China due to a lack of knowledge about the efficient vector capacity to sustain imported $P$. falciparum for the re-establishment of transmission. A foci investigation consists of an assessment of potential Anopheles breeding sites, the collection of adult mosquitoes to identify the species responsible for transmission, and the assessment of the vector's susceptibility to insecticides; these steps are absent in routine surveillance work, but they are required by national guidelines. In addition, 2 malaria-positive patients who were screened by RACD revealed an insensitivity for passive case detection, this insensitivity is a particularly difficult issue for some regions where transmission was absent for several years, such as Hainan, where there was no transmission in 2011. However, in 2015, 7 indigenous cases were reported in this province [24]. This resurgence shows the significance of surveillance systems during the elimination stage, and especially in the post-elimination stage, to monitor for the potential re-introduction of malaria into these areas.

The active foci response required a strategy that combined RACD with vector control to clear the foci [25]. Given proof that asymptomatic people are present at the China-Myanmar border [26], a more sensitive technology is required to screen local residents and frequently mobile populations, including temporary workers and illegal immigrants, who may not routinely use established health services. In China, RACD was performed in the households where a case was identified and neighboring households within a 300-m radius if the focus was considered large (an entire village) [27]. The RDT was 
Table 5 Evaluation of foci response for the 3 types of foci in China

\begin{tabular}{|c|c|c|c|}
\hline \multirow[t]{2}{*}{ Response } & \multicolumn{3}{|c|}{ Foci classification } \\
\hline & Active $^{a}$ & Residual non-active ${ }^{b}$ & Cleared-up \\
\hline Total cases & 28 & 101 & 21 \\
\hline Population at risk ${ }^{c}$ & 6452 & 46,809 & 10,006 \\
\hline No. of foci responding within 7 days (\%) & $28(100)$ & $81(80.2)$ & $1(4.8)$ \\
\hline No. of foci that carried out RACD (\%) & $26(92.9)$ & $84(83.2)$ & $16(76.2)$ \\
\hline Screened population & 1447 & 2985 & 712 \\
\hline No. of malaria-positive patients & 2 & 4 & 0 \\
\hline No. of foci that carried out entomological investigations (\%) & $21(75)$ & $79(78.2)$ & $19(90.5)$ \\
\hline No. of foci that carried out IRS & $26(92.9)$ & $84(83.2)$ & $18(85.7)$ \\
\hline
\end{tabular}

${ }^{a}$ All the indigenous cases reported were considered active foci. In 2015, 28 indigenous cases were reported, and foci response was carried out for all of them ${ }^{\mathrm{b}} \mathrm{A}$ total of 101 cases reported from 2013 to 2014 were categorized as residual non-active foci

'The population at risk represents the recorded data for each type of foci

adopted in the field and all blood samples were taken and sent to the provincial $\mathrm{CDC}$ or national $\mathrm{CDC}$ for PCR verification. Currently, the CDC staff at the ChinaMyanmar border use a high-throughput, low-cost, and highly sensitive screening method based on $18 \mathrm{~S}$ ribosomal RNA to detect asymptomatic sub-patient infections. In 96-well plates, the samples are quantified by the amount of ligated probes that bind continuously to the $18 \mathrm{~S}$ rRNA of the genus Plasmodium; this method may offer an alternative for sensitive, large-scale molecular screening that can be used for RACD [28].

All the measures implemented in these foci, e.g., RACD, IRS, targeted mass drug administration (tMDA), and health education, should follow the national guidelines because they significantly control the foci in the next year. Malaria elimination programs follow-up malaria cases reported by health facilities in order to carry out case investigations that will determine the origin of the infection, whether it has been imported or is due to local malaria transmission. All the active foci should carry out RACD and IRS to reduce the reservoir of asymptomatic and low-density infections, and prevent mosquitoes from biting to block transmission. Targeted interventions such as tMDA may be considered for patients, tMDA was performed on a smaller scale, used for close contacts, households, and villages of index cases.

The residual non-active foci in China cover 19 counties in 3 provinces. Not all the foci investigated and/or responded within 7 days because of poor transportation in Yunnan and Tibet, which caused delays for the CDC staff. To solve this problem, the government has improved road infrastructure; thus, the CDC staff could arrive at the foci in a timely manner. For remote areas, provincial CDC staff designated a central location at which they carry out to carry out case diagnosis, investigation, and treatment. For example, Yunnan Institute of Parasitic Diseases has set up a work station in Nabang village (one village of Yingjiang County, neighboring
Myanmar) to conduct case diagnosis by use of PCR technology during the transmission season.

Despite a lack of indigenous cases occurring at these foci, there is still the possibility of relapse for P. vivax and $P$. ovale. A relapse would require that the local CDC staff emphasize the quality of treatment when visiting each house at the time of radical treatment. An attempt should be made to contact people who were absent during the healthcare visit. Although it is hard to carry out in the primary health care sectors, especially with minority populations who were present at the ChinaMyanmar border and in Tibet, the use of glucose-6phosphate dehydrogenase deficiency assays should also be considered. In China, people who relapsed with $P$. vivax and $P$. ovale should undergo directly observed therapy, taking primaquine for radical treatment [29]. After 1 or 2 years without evidence of transmission, the 72 residual non-active foci may be re-categorized as cleared-up foci.

In addition, we have also summarized the similarities and differences between the frameworks of the WHO and China. While both frameworks could guide classification of foci, WHO's definition and identification of foci mainly classifies foci by tracking them over the last 3 years, and China investigates the response of foci to any current cases, including imported cases. A natural village with any reported cases was assumed to be a focus and was classified based on the Plasmodium species, season, and vectors. Once an imported case was reported, it was checked by microscopy or PCR in a reference lab to determine the Plasmodium species, and then the case was investigated to determine whether it occurred during transmission season. For example, if imported $P$. vivax was reported during transmission season, the CDC staff would carry out a response appropriate for the type of focus, including RACD and IRS, combined with tMDA if it was an active focus. Unlike the WHO classification, the purpose of foci classification 
in China is to allow the CDC staff to determine the appropriate response so that each patient can be identified early and obtain appropriate treatment.

\section{Limitations}

Firstly, not all indigenous cases were well recorded for the exact epidemiological information, especially for domestically mobile (within the country) cases; in this study we missed 60 indigenous cases information. Secondly, the 2 web-based reporting systems were set up in different years, which led to a mismatch in the cases posted in these systems. This issue is also a problem with the PDIRMS, which was established in 2012. More time should be invested in integrating the IDIRMS data with the PDIRMS data and in training local CDC staff. Thirdly, we did not know the exact reasons for the delay in foci investigation and response though these are relatively minimal, they still need specific correction. Fourthly, because the ArcGis software used for foci mapping at village level is not presently available, and because the presentation of all foci in one map would give a low-resolution picture due to the number of foci (more than 200 foci in 2012 and dozens between 2013 and 2015), in this report we provided the indigenous cases at county level from 2010 to 2015.

\section{Conclusion}

This study used nationwide data to categorize foci in China and evaluate the response of these areas during the control and elimination phases. Our approach stratifies future control responses by identifying those locations where the elimination of endemic transmission is needed, such as in the counties at the China-Myanmar border and in Tibet. In addition, this study will help local CDC staff to reassess their needs and responses against different types of foci during the elimination and post-elimination phases.

\section{Abbreviations}

ACF: Residual auto correlation function; CDC: Center for Disease Control and Prevention; IDIRMS: Infectious Diseases Information Reporting Management System; IRS: Indoor residual spraying; LLTN: Long-lasting insecticidal nets; NMEAP: National Malaria Elimination Action Plan; PACF: Residual partial correlation function; PCR: Polymerase chain reaction; PDIRMS: Parasitic Diseases Information Reporting Management System; RACD: Reactive case detection; tMDA: targeted mass drug administration; WHO: World Health Organization

\section{Acknowledgements}

We thank all the staffs in the provincial CDC in China. We also thank LetPub (www.letpub.com) for its linguistic assistance during the preparation of this manuscript.

\section{Funding}

This study was supported National Natural Science Foundation of China (Grant No. 81602904).

\section{Availability of data and materials}

All individual cases in this study could be traced through the Infectious Diseases Information Reporting Management System (IDIRMS, http:// chinacdc.cn) or the Parasitic Diseases Information Reporting Management System (PDIRMS, http://chinacdc.cn).

\section{Authors' contributions}

JF and HT conceived the study and drafted the manuscript. LZ, SSZ (Shaosen Zhang), and ZGX analyzed the data and provided suggestions for improving the quality of the data. HT undertook statistical analysis and model construction. JS carried out the ArcGIS software mapping and data exportation. SSZ (Shuisen Zhou) initiated the study and made major contributions to drafting the manuscript. All authors contributed to the writing of the manuscript and approved the submitted version of the manuscript. All authors read and approved the final manuscript.

\section{Ethics approval and consent to participate}

The data of this study was collected from web-source, so the ethics and participatory consent was not required, and this was approved by National Institute of Parasitic Diseases, Chinese Center for Diseases Control and Prevention.

\section{Consent for publication}

Not applicable.

\section{Competing interests}

The authors declare that they have no competing interests.

\section{Publisher's Note}

Springer Nature remains neutral with regard to jurisdictional claims in published maps and institutional affiliations.

Received: 15 May 2017 Accepted: 28 February 2018

Published online: 07 March 2018

References

1. Ministry of Health: National Malaria Elimination Action Plan. Beijing: Ministry of Health; 2010.

2. Zhou SS, Zhang SS, Zhang L, Rietveld AE, Ramsay AR, Zachariah R, et al. China's 1-3-7 surveillance and response strategy for malaria elimination: is case reporting, investigation and foci response happening according to plan? Infect Dis Poverty. 2015;4:55.

3. Feng J, Xiao H, Xia Z, Zhang L, Xiao N. Analysis of malaria epidemiological characteristics in the People's Republic of China, 2004-2013. Am J Trop Med Hyg. 2015;93(2):293-9.

4. Hu T, Liu YB, Zhang SS, Xia ZG, Zhou SS, Yan J, et al. Shrinking the malaria map in China: measuring the progress of the National Malaria Elimination Programme. Infect Dis Poverty. 2016;5(1):52.

5. Bousema T, Drakeley C, Gesase S, Hashim R, Magesa S, Mosha F, et al. Identification of hot spots of malaria transmission for targeted malaria control. J Infect Dis. 2010;201(11):1764-74.

6. World Health Organization: A framework for malaria elimination. Geneva: World Health Organization; 2017.

7. Zhou SS, Wang Y, Li Y. Malaria situation in the People's Republic of China in 2010. Chin J Parasitol Parasit Dis. 2011;29(6):401-3.

8. Xia ZG, Yang MN, Zhou SS. Malaria situation in the People's Republic of China in 2011. Chin J Parasitol Parasit Dis. 2012;30(6):419-22.

9. Xia ZG, Feng J, Zhou SS. Malaria situation in the People's Republic of China in 2012. Chin J Parasitol Parasit Dis. 2013;31(6):413-8.

10. Zhang L, Feng J, Xia ZG. Malaria situation in the People's Republic of China in 2013. Chin J Parasitol Parasit Dis. 2014;32(6):407-13.

11. Zhang L, Zhou SS, Feng J, Fang W, Xia ZG. Malaria situation in the People's republic of China in 2014. Chin J Parasitol Parasit Dis. 2015;33(5):319-26.

12. Zhang L, Feng J, Zhang SS, Xia ZG, Zhou SS. Malaria situation in the People' s republic of China in 2015. Chin J Parasitol Parasit Dis. 2016:34(6):477-81.

13. Wang L, Wang Y, Jin S, Wu Z, Chin DP, Koplan JP, Wilson ME. Emergence and control of infectious diseases in China. Lancet. 2008:372(9649):1598-605.

14. Cao J, Sturrock HJ, Cotter C, Zhou S, Zhou H, Liu Y, et al. Communicating and monitoring surveillance and response activities for malaria elimination: China's "1-3-7" strategy. PLoS Med. 2014;11(5):e1001642. 
15. Feng J, Yan H, Feng XY, Zhang L, Li M, Xia ZG, et al. Imported malaria in China, 2012. Emerg Infect Dis. 2014;20(10):1778-80.

16. Hay SI, Smith DL, Snow RW. Measuring malaria endemicity from intense to interrupted transmission. Lancet Infect Dis. 2008;8(6):369-78.

17. Clements AC, Barnett AG, Cheng ZW, Snow RW, Zhou HN. Space-time variation of malaria incidence in Yunnan province, China. Malar J. 2009; 8:180.

18. Huang JX, Xia ZG, Zhou SS, Pu XJ, Hu MG, Huang DC, et al. Spatio-temporal analysis of malaria vectors in national malaria surveillance sites in China. Parasit Vectors. 2015;8:146.

19. Moore SJ, Min X, Hill N, Jones C, Zaixing Z, Cameron MM. Border malaria in China: knowledge and use of personal protection by minority populations and implications for malaria control: a questionnaire-based survey. BMC Public Health. 2008;8:344.

20. Li S, Yin S, Wang J, Li X, Feng J. Shifting from control to elimination: analysis of malaria epidemiological characteristics in Tengchong County around China-Myanmar border, 2005-2014. Malar J. 2016;15:45.

21. Xu JW, Li Y, Yang HL, Zhang J, Zhang ZX, Yang YM, et al. Malaria control along China-Myanmar border during 2007-2013: an integrated impact evaluation. Infect Dis Poverty. 2016;5(1):75.

22. Feng J, Liu J, Feng $X$, Zhang L, Xiao H, Xia Z. Towards malaria elimination: monitoring and evaluation of the "1-3-7" approach at the China-Myanmar border. Am J Trop Med Hyg. 2016;95(4):806-10.

23. Duan YZ, Li SG, Kang XH, Yin SQ, XD S. A point-like outbreak caused by secondary transmission from an imported malaria vivax case. Int J Med Parasit Dis. 2013;40:57-9.

24. Lin C, Chen Z, Wang SQ, Luo PZ, Wu DL, Zhen AJ, et al. Investigation of a rare local epidemic of plasmodium malariae infection in Sanya City. Hainan Province Chin Trop Med. 2016;16(5):481-4.

25. Smith Gueye C, Sanders KC, Galappaththy GN, Rundi C, Tobgay T, Sovannaroth $\mathrm{S}$, et al. Active case detection for malaria elimination: a survey among Asia Pacific countries. Malar J. 2013;12:358.

26. Zhao Y, Zhao Y, Lv Y, Liu F, Wang Q, Li P, et al. Comparison of methods for detecting asymptomatic malaria infections in the China-Myanmar border area. Malar J. 2017;16(1):159.

27. Xiao HH, Liu J, Feng J, Zhang SS, Jiang WK, Xia ZG, et al. Screening radius of active case detection and the malaria parasite rate of carriers in ChinaMyanmar border. Chin J Parasitol Parasit Dis. 2015;33(2):86-90.

28. Cheng Z, Wang D, Tian X, Sun Y, Sun X, Xiao N, et al. Capture and ligation probe-PCR (CLIP-PCR) for molecular screening, with application to active malaria surveillance for elimination. Clin Chem. 2015;61(1):821-8.

29. National Health and Family Planning Commission: Technical regulations for application of antimalarials. Beijing: National Health and Family Planning Commission; 2016

\section{Submit your next manuscript to BioMed Central and we will help you at every step:}

- We accept pre-submission inquiries

- Our selector tool helps you to find the most relevant journal

- We provide round the clock customer support

- Convenient online submission

- Thorough peer review

- Inclusion in PubMed and all major indexing services

- Maximum visibility for your research

Submit your manuscript at www.biomedcentral.com/submit

C) Biomed Central 